\title{
Effect of Lattice Size on the Critical Temperature of Ising Nanofilms and Nanotubes on the Square Lattice: Cellular Automata Simulation
}

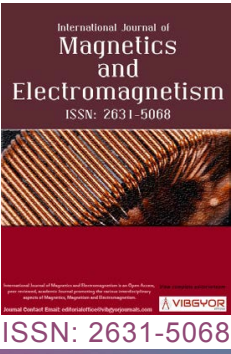

\section{Nabi Javadi}

Faculty of Science, Imam Hossein University, Tehran, Iran

\begin{abstract}
Lattice size dependency of critical temperature of Ising nanofilms and nanotubes on the square lattice have been investigated Using Cellular Automata simulation approach. The obtained values of critical temperature for single-layer nanofilm on the infinite size of lattice is equal to 2.36 , the corresponding value for single-layer nanofilm on the honeycomb lattice was 1.45 . The effects of length and diameter of nanotube on the critical temperature were also studied. For equal size lattices, the critical temperature values of nanotube are larger than those for nanofilm. The critical temperature of a single-layer nanotube on the infinite size lattice was calculated as 2.37 . The proximity of the values for the critical temperature of nanofilm and nanotube in the infinite size of lattice reflects the fact that by increasing the length and diameter of the nanotube, its magnetic behavior tends to be as nanofilm. Such studies seems to be of particular importance in providing tabulated data on the effect of lattice size on critical temperature and magnetic behavior of magnetic nanomaterials.
\end{abstract}

\section{Keywords}

Ising model, Critical temperature, Cellular automata, Magnetic nanofilms, Magnetic nanotubes, Square lattice

\section{Introduction}

Multiple researches have been devoted to the investigation of magnetic nanomaterials, due to the potential applications of these materials in a variety areas such as magnetic recording media, chemical separation, medical diagnosis, and so on [1-3].

Size dependency of phase diagrams and magnetic properties of magnetic nanoparticles have been investigated experimentally [4-9]. Results of these studies show that these materials exhibit some interesting properties for sizes smaller than a certain limit. Research in this field show that a new attitude emerged on the study of nanoscale magnetic materials.

Different theoretical and numerical approaches such as Effective Field Theory (EFT), Monte Carlo, and Cellular Automata (CA) have been used to provide well-tabulated data concerning magnetic properties of magnetic nanostructures [10-19]. Ising model is one of the best methods for modeling and theoretical study of magnetic materials. Here, the effect of the size and shape on the critical

*Corresponding author: Nabi Javadi, Faculty of Science, Imam Hossein University, P. O. Box 1698715861, Tehran, Iran

Accepted: June 04, 2019; Published: June 06, 2019

Copyright: (C) 2019 Javadi N. This is an open-access article distributed under the terms of the Creative Commons Attribution License, which permits unrestricted use, distribution, and reproduction in any medium, provided the original author and source are credited.

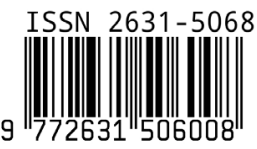

Javadi. Int J Magnetics Electromagnetism 2019, 5:020 
temperature of the Ising nanofilm and nanotube on the square lattice have been investigated by the CA method. The result of the CA method for critical temperature of two-dimensional nanofilm at infinite size of lattice $\left(K_{c}=2.36\right)$ is in accordance with the result of accurate solution by the Onsager $\left(K_{c}=2.27\right)$ [20], which implies the accuracy and reliability of the CA method. So far, this subject, the effect of the size and shape on the critical temperature of the Ising nanofilm and nanotube on the square lattice, from the perspective in our work has not been studied, and these results are reported for the first time. The CA method is a simple, fast, and precise method which have used in many cases for studying the Ising model $[17,19]$.

In a recent paper [18], the size and shape dependencies of phase diagram for Ising nanofilm and nanotube on the honeycomb lattice are investigated. In this work, attempt has been made to obtain the accurate tabulated data for size and shape dependencies of critical temperature of Ising nanofilm and nanotube on the square lattice, and also comparing the obtained data with the results of honeycomb lattice.

\section{Model and Formalism}

In this work the magnetic nanofilms and nanotubes on the square lattice have been investigated. The number of spins on the length and width (diameter) directions of the lattice are shown by $I_{x}$ and $I_{y^{\prime}}$ respectively. $I_{x} I_{y}$ representing the total number of spins in the lattice. Spin variable $s(i, j)= \pm 1$, where $i=1, \ldots, l_{x}$ and $j=1, \ldots, l_{y}$, represents the spin state of $(i, j)$ site in the lattice. Figure 1 , represents the nanofilm on the square lattice. In order to study the magnetic properties of nanotube, the boundary conditions as $s(i, 0)=s\left(i, l_{y}\right)$ and $s\left(i, l_{y}+1\right)=s(i, 1)$ are applied along the $l_{y}$ axis of Figure 1. A square lattice in isotropic case was investigated, $J_{x}=J_{y}$, where $J_{x}$ and $J_{y}$ representing the nearest-neighbor coupling in the $I_{x}$ and $I_{y}$ directions. To updating the lattice sites, the Glauber algorithm with checkerboard techniques are used $[17,18]$. For this purpose, the sites of lattice were presented by black and white colors, and at each step of the simulation, the white sites will be updated firstly, before the black sites. For each lattice site, the probability that the spin state is up $(+1)$, $P_{i, j}^{+}$, is calculated as follows:

$$
P_{i, j}^{+}=\frac{\exp \left(-\beta H_{i, j}^{+}\right)}{\exp \left(-\beta H_{i, j}^{+}\right)+\exp \left(-\beta H_{i, j}^{-}\right)}
$$

Where $\beta=1 / k_{B} T, k_{B}$ and $\mathrm{T}$ are Boltzmann constant and absolute temperature, respectively. The Hamilton for each lattice site is presented as follows:

$H_{i, j}^{ \pm}= \pm\left\{-J_{x}[s(i, j-1)+s(i-1, j)+s(i+1, j)+s(i, j+1)]\right\}$

It is obvious that for each lattice site, the probability that a given spin to be down is

- The average magnetization of the lattice is calculated as follows $[17,18]$ :

$$
<M>=<\sum_{i=1}^{l_{y}} \sum_{j=1}^{l_{y}} s(i, j)>
$$

The average of magnetization per site is calculated as $<m>=<M>/ N$. In order to increase of accuracy of calculations, in each time step of simulation, the average of magnetization per site is calculated over 200 different samples, as independent computer experiment. In this stage the standard error of mean magnetization is in the order of $10^{-6}$.

\section{Results}

In this paper, for calculation of critical temperature, the described method in references $[17,18]$ is used. In this method, which uses the graph of mean magnetization per site as a function of time for a given temperature, the temperature at which

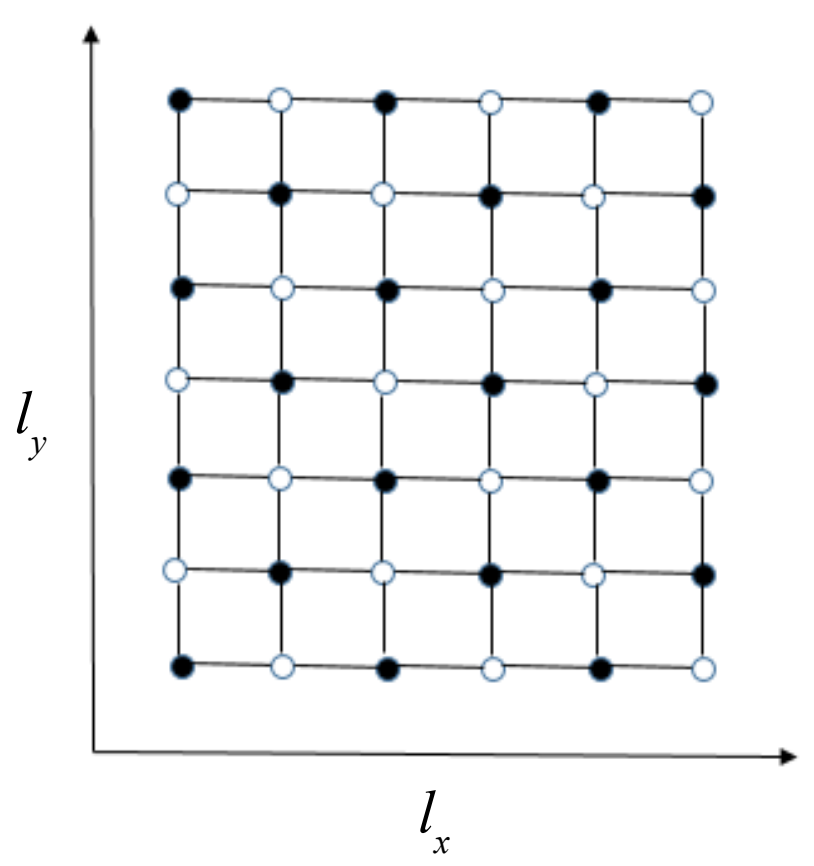

Figure 1: Representation of single-layer Ising nanofilm on the square lattice. 


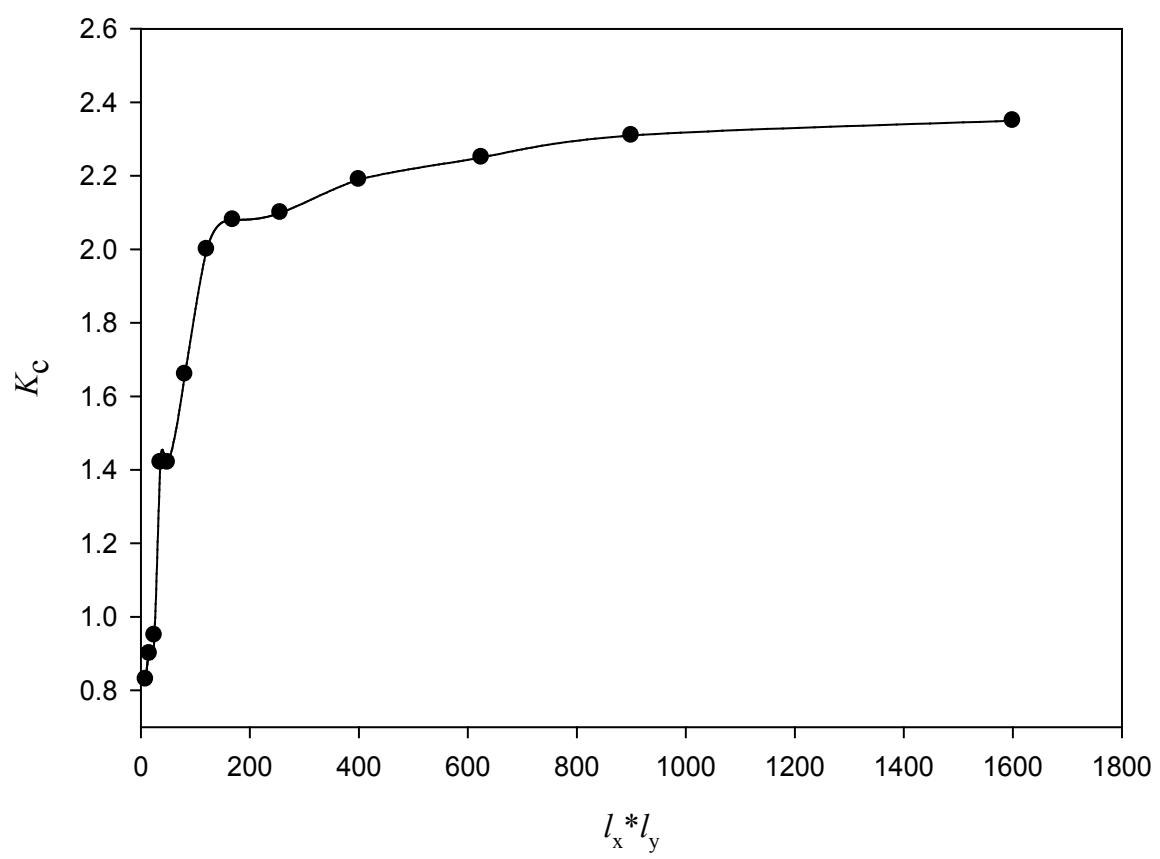

Figure 2: The values of critical temperature versus size of single layer nanofilm on the square lattice.

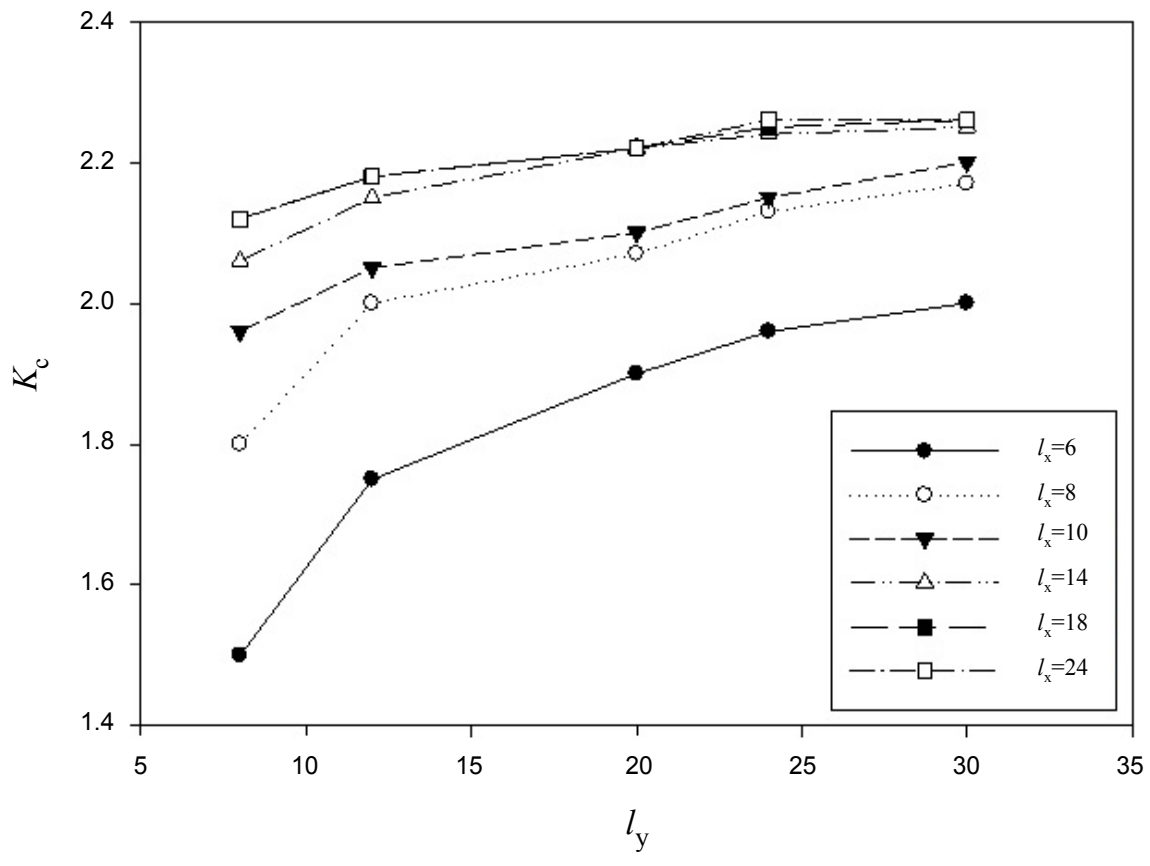

Figure 3: The effect of diameter on the critical temperature of single-layer nanotube on the square lattice.

the ordered magnetic moments (ferromagnetic), change and become disordered (paramagnetic), will be considered as critical temperature.

Figure 2 shows the critical temperature variation in terms of lattice size for a single layer nanofilm on the square lattice. The size of the lattice increases simultaneously with the increase in the length and width of the lattice. As can be seen, with increasing lattice size, the $K_{c}$ value also increased, following a steep slope, close to $l_{x} * l_{y}=200$. Comparison of Figure 2 with Figure 3 of reference [18] shows that the increasing trend of $K_{c}$ for honeycomb lattice up to the size of 500 has been steep. In order to calculate the critical temperature value at infinite size of single-layer nanofilms on the square lattice and also compare the obtained result with 


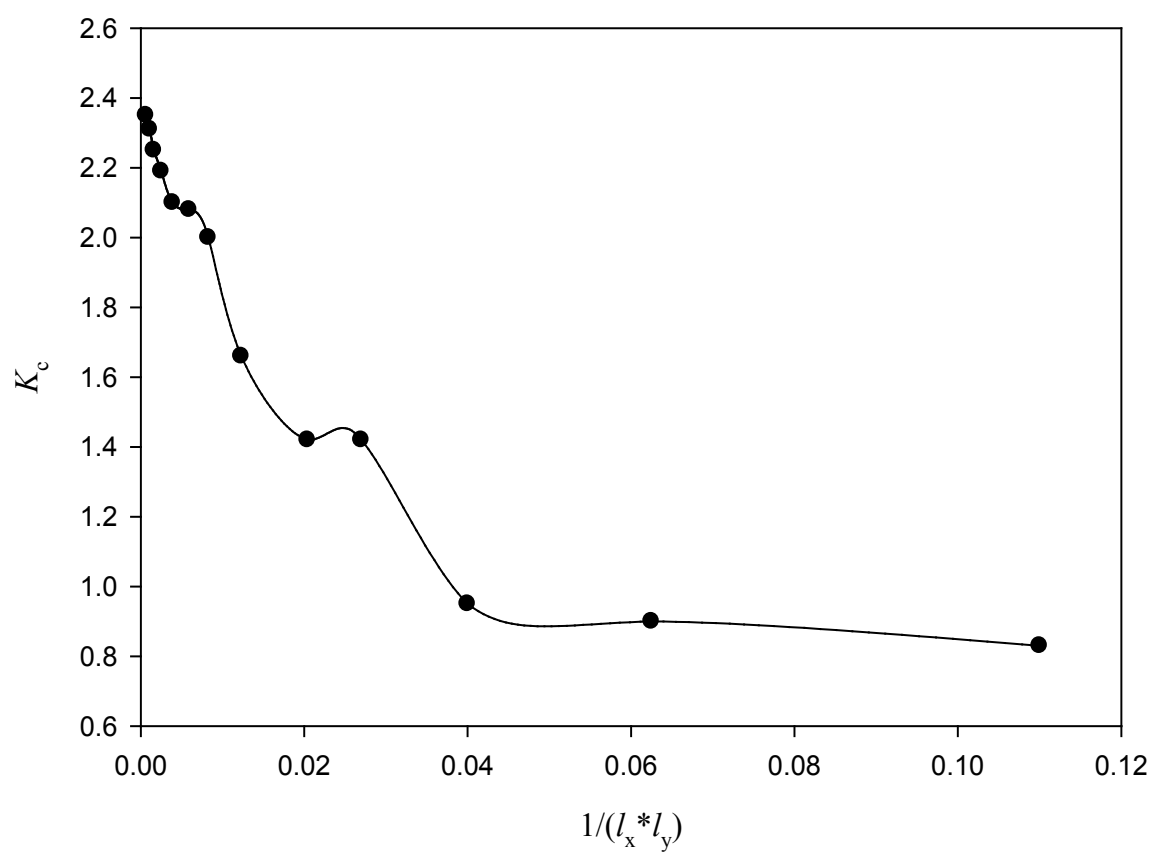

Figure 4: The values of critical temperature versus $x=1 /\left(l_{x} * l_{y}\right)$ of single layer nanofilm on the square lattice.

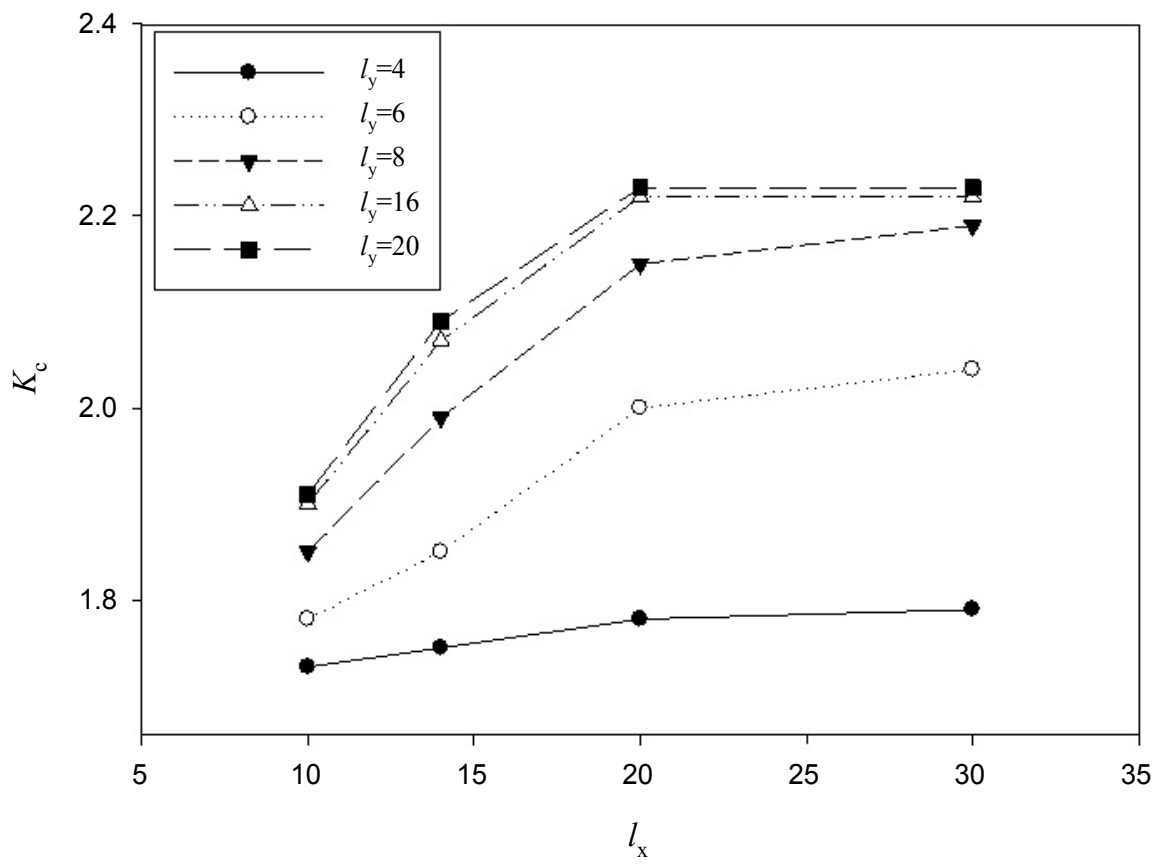

Figure 5: The effect of length on the critical temperature of single-layer nanotube on the square lattice.

the corresponding value for single-layer nanotube, using cure fitting module of Sigma Plot software, the data extrapolation technique was used. To calculate the $K_{c}$ value in infinite size of a single-layer nanofilm, the graph of $K_{c}$ was plotted versus $1 /\left(l_{x} * l_{y}\right)$, and this graph was fitted to a third-order equation (Figure 4 ), as follow:

$$
K_{c}=b_{0}+b_{1} x+b_{2} x^{2}+b_{3} x^{3}
$$

Where $b_{0}=2.36, b_{1}=-57.59, b_{2}=736.26$, $b_{3}=-3080.61$, and $x=1 /\left(l_{x} * l_{y}\right)$. Obviously, by increasing the size of the lattice, the value of the $x$ is decreased so that for lattice with very large sizes, it can be ignored. Therefore, the value of $b_{0}$ is considered as a $K_{c}$ for single-layer nanofilm with infinite lattice size. The corresponding value of $K_{c}$ for single layer nanofilm on the honeycomb lattice 
is 1.54 .

Figure 3 shows the variation trend of $K_{c}$ versus diameter for single-layer nanotube on the square lattice. It can be seen that by increasing the diameter of nanotube, the values of $K_{c}$ is increased and this increasing trend was accentuated for small sizes of lengths. In fact at large lengths, by more increasing of $l_{y}$, the values of $K_{c}$ has few change. Figure 5 shows the effect of length on the critical temperature for single-layer nanotube in different diameter values. Here it is also seen that the values of $K_{c}$ are increased by increasing $l_{x}$. The noteworthy matter of Figure 5 in comparison to Figure 3 is that the variation slope of $K_{c}$ versus length for larger diameters is more. The variation trend of the $K_{c}$ versus $l_{y}$ for single-layer nanofilm and nanotube on the square lattice in equal length $l_{x}=20$ , is shown in Figure 6. As seen from Figure 6, the values of $K_{c}$ for single layer nanofilm and nanotube on the square lattice are larger than the corresponding values on the honeycomb lattice [18]. In honeycomb lattice, the values of $K_{c}$ for nanotube are greater than those for the nanofilm, but in large sizes of lattice, this difference disappears. Also in square lattice, the values of $K_{c}$ for nanotube are initially greater than those for the nanofilm, but with size increasing up to 36 , this difference is not diminished and remains almost constant. For square lattice like the honeycomb, it is expected that in infinite lattice size, the values of $K_{c}$ for two nanosystems are equal. In order to calculate the critical temperature of single-layer nanotube in infinite size of lattice, the curve of $K_{c}$ was plotted versus $1 / l_{y}$, at $l_{x}=24$. Then using extrapolation technique, the value of $K_{c}$ is obtained as 2.37. The proximity of $K_{c}$ for nanofilm and nanotube in infinite size of lattice reflects the fact that by increasing the length and diameter of nanotube, its magnetic behavior tends to be as nanofilm.

\section{Conclusion}

The effect of lattice size on the critical temperature of Ising nanofilms and nanotubes on the square lattice using Cellular Automata approach have been investigated. Thus, the lattice size dependency of critical temperature for single layer nanofilm on the square lattice was evaluated. Using the curve fitting module of SigmaPlot software, the values of $K_{c}$ for single layer nanofilm with infinite size of lattice on the square lattice is obtained as 2.36. The corresponding value of $K_{c}$ for honeycomb lattice is 1.54. For single-layer nanotube on the square lattice, the effects of length and diameter of nanotube on the critical temperature were studied. For square lattice in same lattice sizes, the values of $K_{c}$ for nanotube are larger than the nanofilm. The values of $K_{c}$ for single-layer nanotube in infinite size

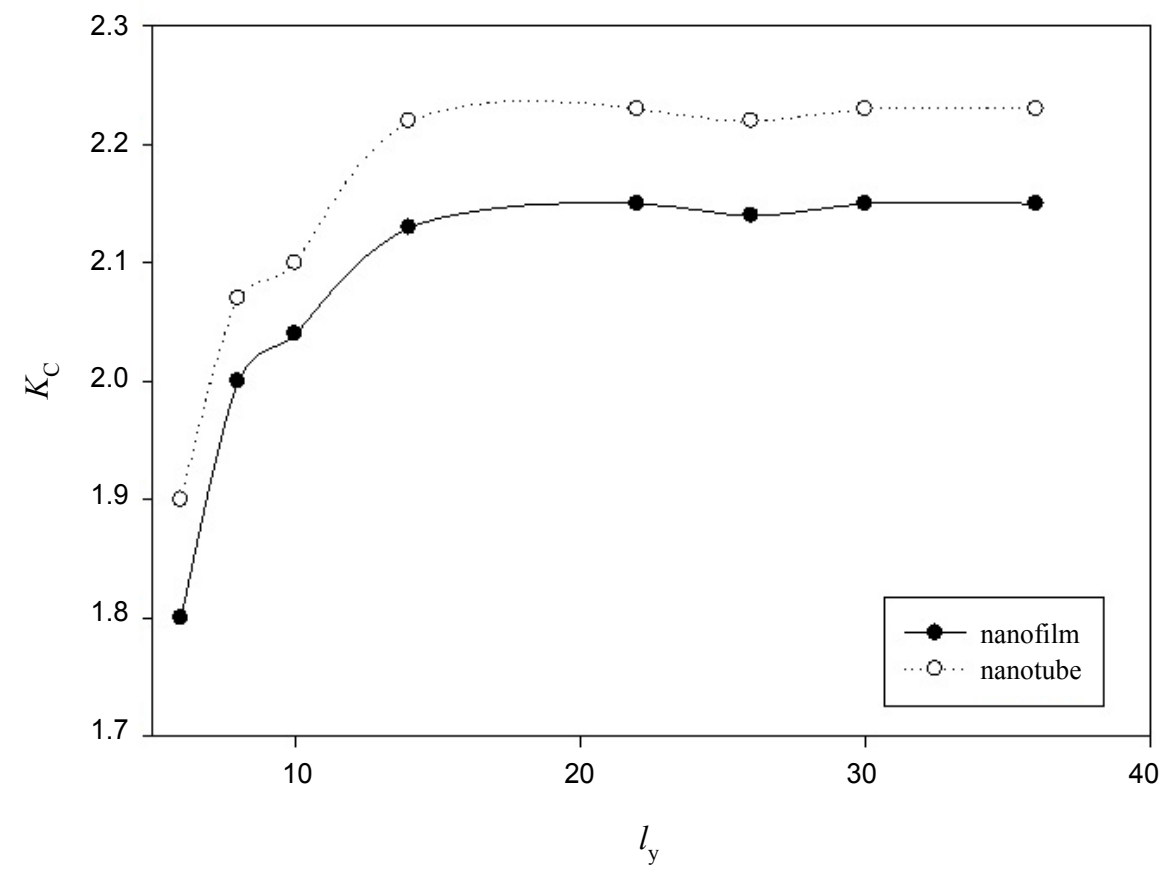

Figure 6: The values of critical temperature versus $\mathrm{I}_{\mathrm{y}}$ for single layer nanofilm and nanotube on the square lattice. 
of lattice was calculated as 2.37 . The proximity of $K_{c}$ for nanofilm and nanotube in infinite size of lattice reflects this fact that by increasing the length and diameter of nanotube, its magnetic behavior tends to be as nanofilm. It seems that such studies are of particular importance to providing tabulated data on the effects of lattice size on the critical temperature and the magnetic behavior of magnetic nanomaterials.

\section{References}

1. Li C, Ma C, Wang F, Xil Z, Wang Z, et al. (2012) Preparation and biomedical applications of coreshell silica/magnetic nanoparticle composites. J Nanosci Nanotechnol 12: 2964-2972.

2. Mou X, Ali Z, Li S, He N (2015) Applications of magnetic nanoparticles in targeted drug delivery system. J Nanosci Nanotech 15: 54-62.

3. Geng Y (2012) Magnetic nanotubes: Synthesis, properties, and applications. Crit Rev Solid State Mater Sci 37: 75-93.

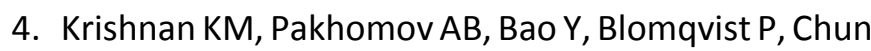
$Y$, et al. (2006) Nanomagnetism and spin electronics: Materials, microstructure and novel properties. J Mater Sci 41: 793-815.

5. Lim CW, Lee IS (2010) Magnetically recyclable nanocatalyst systems for the organic reactions. Nano Today 5: 412-434.

6. Bas JA, Calero JA, Dougan MJ (2003) Sintered soft magnetic materials. Properties and applications. J Magn Magn Mater 254: 391-398.

7. Toshitaka I, Kenichi Y, Kunio A (2016) Effect of Particle Size on the Magnetic Properties of Ni Nanoparticles Synthesized with Trioctylphosphine as the Capping Agent. Nanomaterials (Basel) 6: E172.

8. He X, Zhong W, Au CT, Du Y (2013) Size dependence of the magnetic properties of $\mathrm{Ni}$ nanoparticles prepared by thermal decomposition method. Nanoscale Res Lett 8: 446-555.

9. Koseoglu $Y$, Kavas $H$ (2008) Size and surface effects on magnetic properties of $\mathrm{Fe}_{3} \mathrm{O}_{4}$ nanoparticles. J Nanosci Nanotechnol 8: 584-590.
10.Kaneyoshi T (2012) Compensation point (or points) of nanoscaled transverse Ising thinfilms: Effects of surface dilution. Phase Transit 85: 264-278.

11. Kaneyoshi T (2012) Characteristic phenomena in nanoscaled transverse Ising thinfilms with diluted surfaces. Physica B: Condensed Matter 407: 4358-4364.

12.Kaneyoshi T (2012) Two compensation points induced by surface parameters in nanoscaled transverse Ising ferrimagnetic thinfilms. Solid State Commun 152: 1686-1689.

13.Kaneyoshi T (2013) Phase diagrams in nanoscaled Ising thinfilms with diluted surfaces; effects of interlayer coupling at the surfaces. Physica B: Condensed Matter 408: 126-133.

14. Masrour R, Jabar A, Hamedoun M, Benyoussef A (2016) Magnetic Behavior in Ising Nanoisland: A Monte Carlo Study. J Supercond Nov Magn 29: 24132419.

15. Masrour R, Bahmad L, Hamedoun M, Benyoussef A, Hlil EK (2013) The magnetic properties of a decorated Ising nanotube examined by the use of the Monte Carlo simulations. Solid State Commun 162: 53-56.

16.Vatansever E, Polat HJ (2013) Monte Carlo investigation of a spherical ferrimagnetic core-shell nanoparticle under a time dependent magnetic field. Magn Magn Mater 343: 221-227.

17.Ghaemi M, Javadi N (2017) Construction of phase diagrams for nanoscaled Ising thin films on the honeycomb lattice using cellular automata simulation approach. Phase Transit 90: 1089-1100.

18.Ghaemi M, Javadi N (2018) Investigation of size and shape dependencies of phase diagrams of the Ising nanofilms and nanotubes on the honeycomb lattice using cellular automata simulation approach. Journal of Superconductivity and Novel Magnetism 31: 2421-2429.

19. Astaraki M, Ghaemi M, Afzali K (2018) Investigation of phase diagrams for cylindrical Ising nanotube using cellular automata. Physics letters A 382: 1291-1297.

20.Onsager L (1944) Crystal Statistics. I. A two-dimensional model with an order-disorder transition. Phys Rev 65: 117-149. 